\title{
EVEN PERFECT NUMBERS AND THEIR EULER'S FUNCTION
}

\section{SYED ASADULLA}

\author{
Department of Mathematics and Computing Sciences \\ St. Francis Xavier University \\ Antigonish, Nova Scotia. B2G 100. Canada.
}

(Received February 21, 1985, and in revised form, June 23, 1986)

ABSTRACT. The purpose of this article is to prove some results on even perfect numbers and on their Euler's function. The results obtained are all straightforward deductions from well-known elementary number theory.

KEY WORDS AND PHRASES. Perfect number; triangular number; Euler's function; number of divisors function.

1980 MATHEMATICS SUBJECT CLASSIFICATION CODE. 10A40

\section{INTRODUCTION.}

A positive integer is called a perfect number if it is equal to the sum of its positive divisors excluding itself.

The $n^{\text {th }}$ triangular number is the sum of the first $n$-positive integers

$$
\sum_{k=1}^{n} \mathbf{k}=\frac{1}{2} n(n+1)=T(n)
$$

Euler's function $\phi(n)$ is the number of positive integers less than or equal to $n$ and relatively prime to $n$.

The number of divisors function $d(n)$ is the number of positive divisors of n.

\section{MAIN RESULTS.}

The proof of the following Theorem 1 can be found in many elementary number theory books; see, for example, [1:p. 98].

THEOREM 1. If $n$ is an even perfect number, there exists a prime $2^{p}-1$ such that $\mathrm{n}=2^{\mathrm{p}-1}\left(2^{\mathrm{p}}-1\right)$.

THEOREM 2. If $T\left(p_{1}\right)$ is any even perfect number, where $p_{1}$ is prime, and if $p_{k}$ is the first prime in the sequence $\left\{p_{2}, p_{3}, \ldots, p_{j}, \ldots\right\}$ where $p_{j}=2 p_{j-1}+1$, then $T\left(p_{k}\right)$ is the next even perfect number.

PROOF. It follows from Theorem 1 that an even perfect number is of the form $2^{n-1}\left(2^{n}-1\right)$, where $2^{n}-1$ is prime. Now, $2^{n-1}\left(2^{n}-1\right)$ can be written as $T\left(p_{1}\right)$, where $p_{1}=2^{n}-1$. Let $p_{i}$ be any composite term of the sequence $\left\{p_{2}, p_{3}, \ldots, p_{j}\right.$, $\ldots\}$. It can be shown that $p_{i}=2^{n+i-1}-1$, using the facts $p_{i}=2^{n}-1$, and $p_{j}=2 p_{j-1}+1$. Now, it follows from Theorem 1 that $T\left(p_{i}\right)=2^{n+i-2}\left(2^{n+i-1}-1\right)$ is 
not an even perfect number. Let $p_{k}$ be the first prime in the sequence $\left\{p_{2}, p_{3}\right.$, $\left.\ldots, p_{j}, \ldots\right\}$. As before, $p_{k}=2^{n+k-1}-1$. Observe that $T\left(p_{k}\right)=2^{n+k-2}\left(2^{n+k-1}-1\right)$ is of the form $2^{m-1}\left(2^{m}-1\right)$, where $2^{m}-1$ is prime and thus $T\left(p_{k}\right)$ is an even perfect number by Theorem 1 .

EXAMPLE. $\quad T(3)=\frac{1}{2}(3)(4)=6, \quad T(7)=\frac{1}{2}(7)(8)=28$.

$$
T(31)=\frac{1}{2}(31)(32)=496, T(127)=\frac{1}{2}(127)(128)=8128, \ldots
$$

THEOREM 3. If $n=2^{m-1}\left(2^{m}-1\right)$, then, $n=1^{3}+3^{3}+\ldots+\left[2^{(m+1) / 2}-1\right]^{3}$.

PROOF. Observe that $2^{(m+1) / 2}=2 k$, where $k=2^{(m-1) / 2}$. Now, consider $1^{3}+2^{3}+3^{3}+\ldots+(2 k-1)^{3}+(2 k)^{3}=[1+2+3+\ldots+(2 k-1)+(2 k)]^{2}=\left[\frac{1}{2}(2 k)(2 k+1)\right]^{2}$

which implies that $1^{3}+2^{3}+3^{3}+\ldots+(2 k-1)^{3}$

$$
\begin{aligned}
& =k^{2}(2 k+1)^{2}-\left[2^{3}+4^{3}+\ldots+(2 k)^{3}\right] \\
& =k^{2}(2 k+1)^{2}-2^{3}\left(1^{3}+2^{3}+\ldots+k^{3}\right) \\
& =k^{2}(2 k+1)^{2}-8(1+2+\ldots+k)^{2} \\
& =k^{2}(2 k+1)^{2}-8\left[\frac{1}{2} k(k+1)\right]^{2} \\
& =k^{2}(2 k+1)^{2}-2 k^{2}(k+1)^{2}=k^{2}\left(2 k^{2}-1\right) .
\end{aligned}
$$

Since $k=2^{(m-1) / 2}$, it follows that $1^{3}+3^{3}+\ldots+\left[2^{(m+1) / 2}-1\right]^{3}=2^{m-1}\left(2^{m}-1\right)=n$.

The following Corollary 1 , follows from Theorem 3 .

COROLLARY 1. If $n$ is an even perfect number $2^{p-1}\left(2^{p}-1\right)$, then

$$
n=1^{3}+3^{3}+\ldots+\left[2^{(p+1) / 2}-1\right]^{3} \text {. }
$$

EXAMPLE. $\quad 496=1^{3}+3^{3}+5^{3}+7^{3}: p=5$.

The proof of the following Theorem 4 can also be found in many elementary number theory books; see, for example [1: p. 63].

THEOREM 4. If $n=p_{1}^{\alpha} p_{2}^{\alpha_{2}} \ldots p_{k}^{\alpha}$,

$$
\text { then } \phi(n)=n\left(1-\frac{1}{p_{1}}\right)\left(1-\frac{1}{p_{2}}\right) \ldots\left(1-\frac{1}{p_{k}}\right), \text { where }
$$

$p_{1}, p_{2}, \ldots, p_{k}$ are distinct primes and $\alpha_{1}, \alpha_{2}, \ldots, \alpha_{k}$ are positive integers.

As a consequence of Theorem 4, one can easily obtain Theorem 5, Corollary 2.

and Corollary 3

THEOREM 5. $n=2^{p-1}\left(2^{p}-1\right)$ is an even perfect number if and only if

$$
\phi(n)=2^{p-1}\left(2^{p-1}-1\right) \text {, where } 2^{p-1} \text { is prime. }
$$

COROLLARY 2. If $n$ is an even perfect number, then $\phi(n)=n-4^{p-1}$.

EXAMPLE. $\phi(8128)=\phi\left(2^{6}\right) \phi(127)=4032=8128-4^{6}$.

COROLLARY 3. If $n$ is an even perfect number, then $\phi(n)=\frac{n}{2}-2^{p-2}$.

THEOREM 6. If $n_{1}, n_{2}, \ldots, n_{k}$ are $k$-distinct even perfect numbers,

$$
\text { then } \phi\left(n_{1} n_{2} \ldots n_{k}\right)=2^{k-1} \phi\left(n_{1}\right) \phi\left(n_{2}\right) \ldots \phi\left(n_{k}\right) \text {. }
$$

PROOF. $\quad \phi\left(n_{1} n_{2} \ldots n_{k}\right)$

$$
\begin{aligned}
& =\phi\left[2^{p_{1}-1}\left(2^{p_{1}}-1\right) 2^{p_{2}-1}\left(2^{p_{2}}-1\right) \ldots 2^{p_{k}-1}\left(2^{p_{k}}-1\right)\right] \\
& =\phi\left[2^{p_{1}+p_{2}+\ldots+p_{k}-k}\left(2^{p_{1}}-1\right)\left(2^{p_{2}}-1\right) \ldots\left(2^{p_{k}}-1\right)\right] \\
& =\phi\left(2^{p_{1}+p_{2}+\ldots+p_{k}-k}\right) \phi\left(2^{p_{1}}-1\right) \phi\left(2^{p_{2}}-1\right) \ldots \phi\left(2^{p_{k}}-1\right)
\end{aligned}
$$




$$
\begin{aligned}
& =2^{p_{1}+p_{2}+\ldots+p_{k}-k-1}\left(^{p_{1}}-2\right)\left(2^{p_{2}}-2\right) \ldots\left(2^{p_{k}}-2\right) \\
& \left.=2^{k-1} \cdot 2^{p_{1}-1}\left(2^{p_{1}-1}-1\right) \cdot 2^{p_{2}-1}\left(2^{p_{2}-1}-1\right) \cdot 2^{p_{k}^{-1} p_{k}-1}-1\right) \\
& =2^{k-1} \phi\left(n_{1}\right) \phi\left(n_{2}\right) \ldots \phi\left(n_{k}\right) .
\end{aligned}
$$

The following Theorem 7 is proved in many books on elementary number theory: see, for example, [1: p. 96].

THEOREM 7. If $n=\prod_{i=1}^{k} p_{i}^{\alpha_{i}}$, then $d(n)=\underset{i=1}{k}\left(1+\alpha_{i}\right)$, where $p_{i}, i=1, \ldots$ $k$ are distinct primes and $\alpha_{1}, 1=1, \ldots, k$ are positive integers, and $d(n)$ is the number of divisors function.

THEOREM 8. If $n=\prod_{i=1}^{k} p_{i}^{\alpha_{i}}$, and $d(n)$ is an even perfect number $2^{p-1}\left(2^{p}-1\right)$, then

i) $\quad \mathbf{p} 2 \mathbf{k}$.

ii) $\quad \alpha_{j}=2^{\mu}\left(2^{p}-1\right)-1$ for exactly one $j$ such that $1<j \leq k$ and $\mu_{j} \geq 0$.

iii) $\quad \alpha_{i}=2^{\mu_{i}}-1$, where $\mu_{i}>0,1 \leq i \leq k, i \neq j$.

iv) $\sum_{i=1}^{k} \mu_{i}=p-1$.

PROOF. From Theorem 5, one obtains $d(n)=\underset{i=1}{k}\left(1+\alpha_{i}\right)=2^{p-1}\left(2^{p}-1\right)$, which implies that $\left(2^{p}-1\right)$ divides exactly one of the factors $\left(1+\alpha_{i}\right), 1 \leq i \leq k$, say $\left(1+\alpha_{j}\right)$. Thus $\left(1+\alpha_{j}\right)=\left(2^{p}-1\right) \cdot \lambda$ for some $\lambda$ and exactly one $j$ such that $1 \leq j \leq k$, and $\left(2^{p}-1\right) \cdot \lambda \cdot \prod_{\substack{i=1 \\ i \neq j}}^{k}\left(1+\alpha_{1}\right)=2^{p-1}\left(2^{p}-1\right)$, that is. $\lambda \cdot \prod_{\substack{i=1 \\ i \neq j}}^{k}\left(1+\alpha_{i}\right)=2^{p-1}$, which implies that $1+\alpha_{i}=2^{\mu_{i}}, 1 \leq 1 \leq k$, $1 \neq j, \mu_{i}>0 ; \lambda=2^{\mu_{j}}, \mu_{j} \geq 0$ and $\sum_{i=1}^{k} \mu_{i}=p-1$, which is (iv). Observe that $\sum_{i=1}^{k} \mu_{i}>k-1$ since $\mu_{i}>0$ for $1 \neq j$ and $\mu_{j}>0$. Thus, p-1 $2 k-1$ or $p 2 k$, which is (i). Now, $\left(1+\alpha_{j}\right)=\left(2^{p}-1\right) \cdot \lambda=\left(2^{p}-1\right) \cdot 2^{\mu}$ for exactly one $j$, such that $1 \leq j \leq k$ and $\mu_{j} \geq 0$ implies that $\alpha_{j}=2^{\mu}$. $\left(2^{p}-1\right)-1$ for exactly one $j$ such that $1 \leq j \leq k$ and $\mu_{j} 20$, which proves (ii). 
Finally, $1+\alpha_{i}=2^{\mu_{i}}, \quad 1 \leq i \leq k, \quad i \neq j, \mu_{i}>0$ implies that $\alpha_{i}=2^{\mu_{i}}-1,1 \leq i \leq k, \quad i \neq j, \mu_{i}>0$, which proves (iii).

ACKNOWLEDGEMENT. The author wishes to thank the referee for his many helpful suggestions.

\section{REFERENCES}

1. SHOCKLEY, J.E., Introduction to Number Theory, Holt, Rinehart and Winston, New York, N.Y.: 1967. 


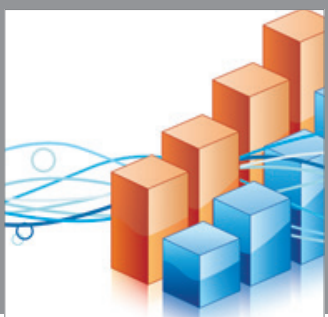

Advances in

Operations Research

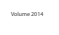

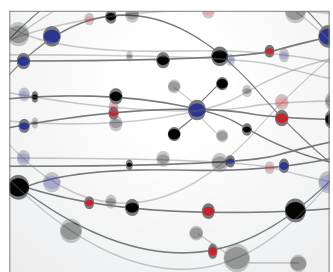

\section{The Scientific} World Journal
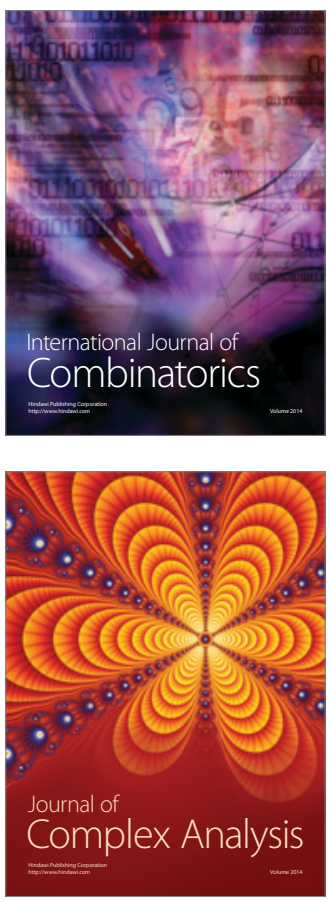

International Journal of

Mathematics and

Mathematical

Sciences
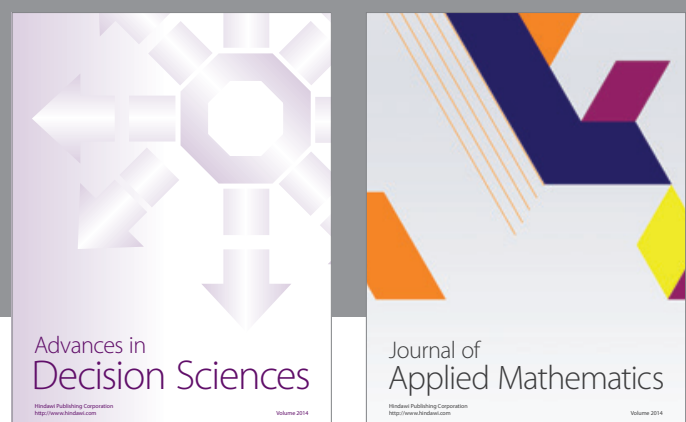

Journal of

Applied Mathematics
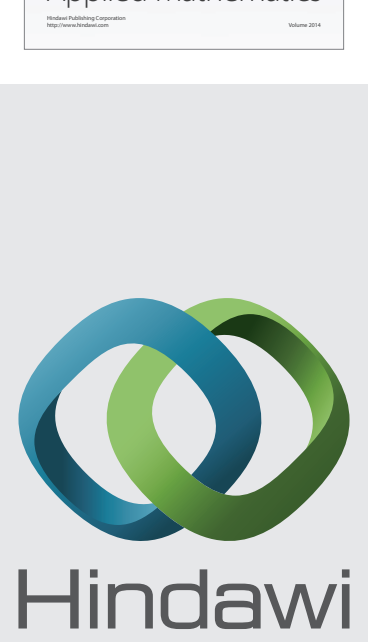

Submit your manuscripts at http://www.hindawi.com
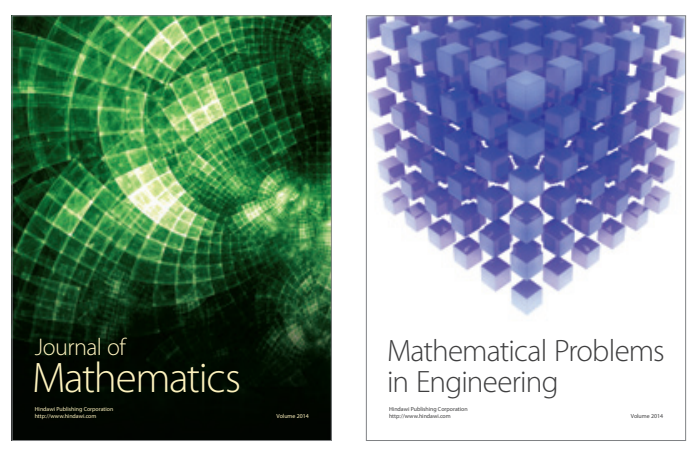

Mathematical Problems in Engineering
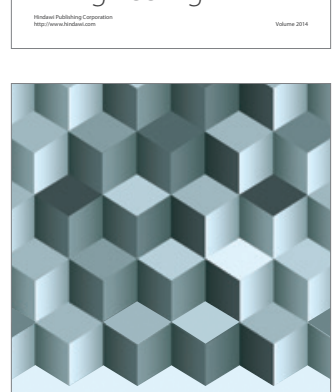

Journal of

Function Spaces
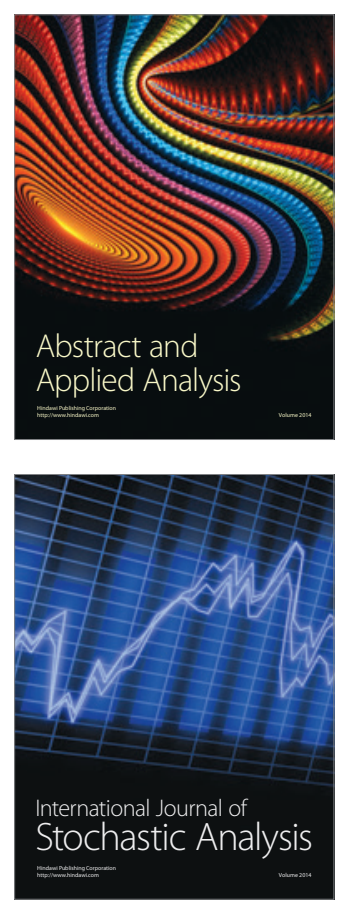

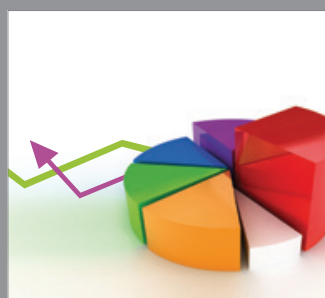

ournal of

Probability and Statistics

Promensencen
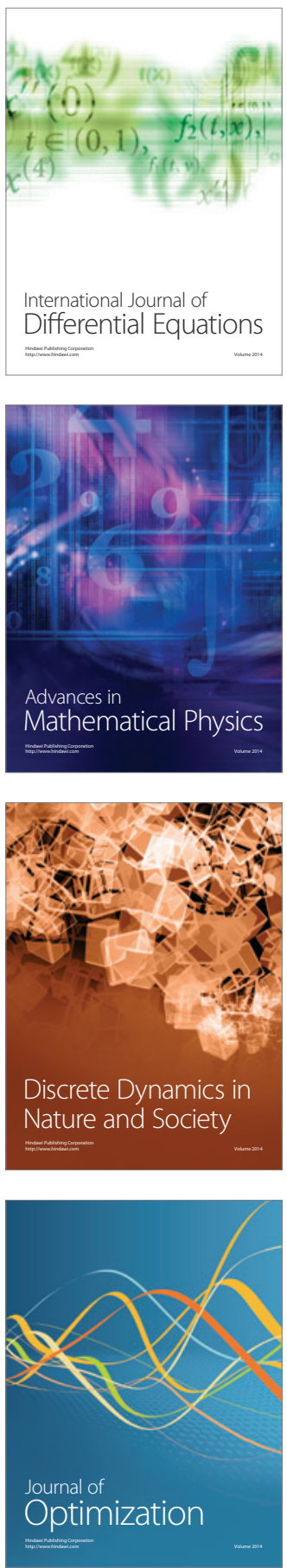